\title{
AUTOCORRELATION FUNCTION AND MUTUAL INFORMATION FROM SHORT EXPERIMENTAL TIME SERIES*
}

\author{
A. ZDUNIAK AND J. ŁUSAKOWSKI \\ Institute of Experimental Physics, University of Warsaw \\ Hoża 69, 00-681 Warszawa, Poland
}

\begin{abstract}
A nonlinear dynamics of self-generated current oscillations in semi-insulating $\mathrm{GaAs}$ was studied by the reconstruction of an attractor from a short (14500 points) time series. Two methods of choosing of a time constant $(\tau)$ for this reconstruction are compared. One of them assumes $\tau$ to be an argument of the first zero of the autocorrelation function and the other takes $\tau$ as an argument of the first minimum of the mutual information. It is shown that for periodic oscillations both methods are equivalent, but for chaotic ones only the mutual information gives a time constant which does not depend on a time series used for calculations.
\end{abstract}

PACS numbers: $05.45 .+b$

\section{Introduction}

One of the ways of analysis of the dynamics of a nonlinear system is based on a reconstruction of its attractor. A widely used method of Grassberger and Procaccia [1] of the reconstruction of a $n$-dimensional projection of an attractor from a scalar time series $x\left(t_{i}\right)$ requires determination of a time delay $\tau$ which is then used for generation of points $X_{i}$ of an $n$-dimensional phase space: $X_{i}=$ $\left(x\left(t_{i}\right), x\left(t_{i}+\tau\right), \ldots, x\left(t_{i}+(n-1) \tau\right)\right)$. It is crucial to choose such a value of $\tau$ which allows to regard the coordinates of a point $X_{i}$ as independent variables. For this purpose one can calculate the autocorrelation function $F$ of the series, and to choose $\tau$ as an argument of the first zero $(f)$ of $F$. Another approach was proposed by Fraser and Swinney who introduced the mutual information $M$ of the signal [2]. A value of $M(\tau)$ says how many bits of information can be obtained about the value of $x\left(t_{i}+\tau\right)$ if the value of $x\left(t_{i}\right)$ is known. An argument corresponding to a minimum of $M$ is thus a good choice for the time delay $\tau$.

*This work is partially supported by the State Committee for Scientific Research (Republic of Poland) grant No. 202659101. 
In the present paper the two methods of determination of $\tau$ are applied to short experimental time series. It is shown that for chaotic oscillations the mutual information in contrast to the autocorrelation function gives a reliable value of $\tau$.

The dynamical system chosen for this study was a sample of semi-insulating (SI) GaAs. At room temperature two types of spontaneous current oscillations are usually observed in this material. For small bias (corresponding to a linear or slightly sublinear part of the current-voltage characteristic [3]) one observes small amplitude oscillations which are caused by a spatio-temporal instability which involves only a limited part of the sample in the vicinity of the negatively biased contact [4]. For larger bias (corresponding to a negative differential conductivity part of the characteristic [5]) large amplitude oscillations are generated which reflect the motion of high electric field domains along the sample. These two types of oscillations will be referred to as type $A$ and $B$, respectively. A transition from one type of oscillations to the other is usually connected with their chaotization.

\section{Experiment and results}

A sample used in the present study was cut from a wafer of undoped SI GaAs. The sample was supplied with Au contacts and connected in series with a
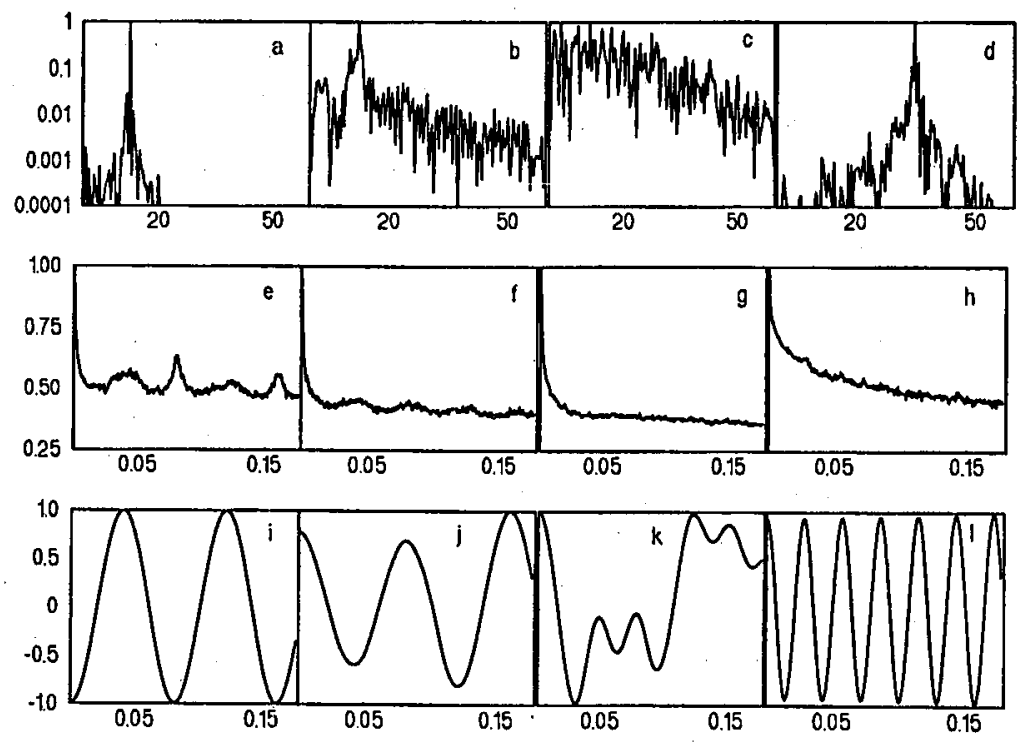

Fig. 1. Power spectra (a-d), the mutual information (e-h) and the autocorrelation function (i-l) of the measured signal. The columns from left to right give the results of calculations based on experimental signals measured at $240 \mathrm{~V}$ (type $A$ oscillations), $310 \mathrm{~V}, 320 \mathrm{~V}$ (chaotic oscillations) and $400 \mathrm{~V}$ (type $B$ oscillations), respectively. 8196 and 12000 subsequent points were taken from each time series for calculations of $F$ and $M$, respectively. The unit on the horizontal axis is equal to $1 \mathrm{~Hz}$ and $0.044 \mathrm{~ms}$ for parts (a)-(d) and (e)-(1), respectively. 

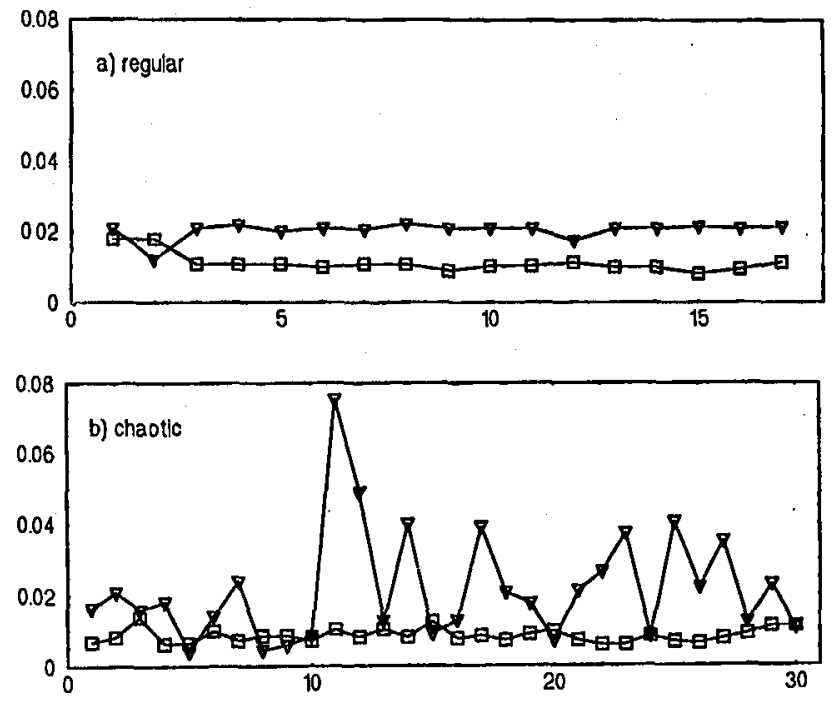

Fig. 2. A position of the first zero of the autocorrelation function (triangles) and the first minimum of the mutual information (squares) for periodic type $A$ (a) and chaotic (b) oscillations, respectively. The unit on the vertical axis is equal to $0.0446 \mathrm{~ms}$. The horizontal axis numbers time series used for calculations.

load resistor and a voltage source. The experiment was performed at a stabilized temperature equal to $305.5 \pm 0.1 \mathrm{~K}$. The sample was shielded from the visible light. Type $A$ oscillations were observed for the bias greater than about $212 \mathrm{~V}$ and those of type $B$ for the bias greater than $370 \mathrm{~V}$. A chaotic signal was observed for voltages between $310 \mathrm{~V}$ and $350 \mathrm{~V}$. In each measurement a 14500-point time series with $0.0446 \mathrm{~ms}$ interval between subsequent points was collected. Seventeen and thirty time series were collected for type $A$ and type $B$ oscillations, respectively. The break between collecting of subsequent series lasted for a few seconds. The mutual information was calculated according to the algorithm proposed by Fraser and Swinney [2] and the autocorrelation function as an inverse of the fast Fourier transform of the time series.

Results of calculations of $M, F$ and power spectra of experimental signals are shown in Fig. 1. Figure 1a-1d shows the highest four orders of magnitude of the normalized power spectra. An increase in the applied bias causes a broadening of the power spectrum of the type $A$ oscillations (Fig. 1a and Fig. 1b). The spectrum takes the shape of a broad-band noise for the chaotic state (Fig. 1c) and shows again a peak-like form when periodic oscillations (of the type $B$ ) are restored (Fig. 1d). Chaotization of the signal is connected with a loss of the periodicity of $F$ (Fig. $1 \mathrm{j}$ and $1 \mathrm{k}$ ).

The main result is shown in Fig. 2. For periodic oscillations both $f$ and $m$ show a small scatter around a mean value, whereas for the chaotic case a position of $f$ shows a large scatter which is not observed for $m$. An oscillatory behaviour of the mutual information vanishes as the signal becomes more and more chaotic 
(Fig. 1e-1g), which is, however, not a general rule [2]. In a fully chaotic state (Fig. 1g) no minimum of $M$ was observed and in this case $m$ was defined as an argument for which the normalized value of $M$ is equal to 0.5 . This value of $M$ corresponds to $m$ determined for the periodic case shown in Fig. 1e. It was verified that choosing of a value different from 0.5 leads also to a repeatable value of $m$. In other words, the shape of the mutual information curve is highly repeatable for all measured series. Thus, the mutual information offers a reliable time constant of any signal, periodic or chaotic, even when it is calculated from a short time series. On the other hand, the autocorrelation function appears to be of no use for the chaotic case.

In conclusion, the mutual information is recommended as a tool which allows to determine a proper time constant for the reconstruction of a chaotic attractor. The presented results show that it can be calculated from the short time series which becomes particularly important when collecting long time series is impossible due to hardware limitations.

\section{Acknowledgments}

The authors are much thankful to Dr. E. Kamińska and Dr. A. Piotrowska, Institute of Electron Technology, Warsaw, for preparing the contacts to the sample and to Prof. M. Grynberg for helpful discussions.

\section{References}

[1] P. Grassberger, I. Procaccia, Physica D 9, 189 (1983).

[2] A.M. Fraser, H.L. Swinney, Phys. Rev. A 33, 1134 (1986).

[3] J. Eusakowski, Mater. Sci. Eng. B 6, 1 (1990).

[4] B. Willing, J.K. Maan, private communication.

[5] U. Rau, J. Peinke, J. Parisi, K. Karpierz, J. Lusakowski, W. Knap, Phys. Lett. A 152, 356 (1991). 